\title{
Avaliação das alterações fundoscópicas na miopia degenerativa
}

\author{
Funduschanges evaluation in degenerative myopia
}

\author{
Oswaldo Ferreira Moura Brasil ${ }^{1}$ \\ Maria Vitoria Oliveira Moura Brasil ${ }^{2}$ \\ Ricardo Mig'uel Japiassú ${ }^{3}$ \\ Ana Luiza Biancardi ${ }^{4}$ \\ Débora Duarte de Souza ${ }^{5}$ \\ Renato Corrêa de Souza Oliveira ${ }^{6}$ \\ Haroldo Vieira de Moraes $\mathrm{Jr}^{7}$
}

\begin{tabular}{|c|}
\hline RESUMO \\
\hline $\begin{array}{l}\text { Objetivo: Avaliar a presença de alterações fundoscópicas em pacientes } \\
\text { portadores de miopia degenerativa. Métodos: Quarenta pacientes com } \\
\text { erro refrativo de pelo menos }-6,00 \text { dioptrias foram selecionados para } \\
\text { exame oftalmológico, complementado por retinografia do pólo posterior } \\
\text { e ecobiometria. Resultados: Foram estudados } 57 \text { olhos de } 37 \text { pacientes } \\
\text { com erro refrativo de }-6,25 \text { a }-28,50 \text { dioptrias, com média de }-14,05 \text {, e } \\
\text { comprimento axial de } 26,06 \text { a } 32,86 \mathrm{~mm} \text {, com média de } 28,01 \text {. Encontramos } \\
\text { crescente temporal em } 36,5 \% \text { e circunferencial em } 21 \% \text { dos olhos. Vasos } \\
\text { da coróide foram visualizados em } 35 \% \text { dos olhos. As alterações do pólo } \\
\text { posterior foram as seguintes: estafiloma posterior em } 10,5 \% \text {, mancha de } \\
\text { Fuchs em } 3,5 \% \text { e "lacquer cracks" em } 1,5 \% \text {. O exame da periferia retiniana } \\
\text { evidenciou atrofia corio-retiniana tipo pavimentosa em } 17,5 \% \text {, branco } \\
\text { sem pressão em } 10,5 \% \text {, degeneração "lattice" em } 5 \% \text {, ruptura retiniana } \\
\text { em } 3,5 \% \text { e retinosquise em } 1,5 \% \text { dos olhos examinados. Conclusões: } \\
\text { Alterações fundoscópicas que levam à baixa visual são freqüentes em } \\
\text { pacientes com miopia degenerativa. O exame da periferia retiniana é muito } \\
\text { importante nestes pacientes devido ao risco aumentado de desco- } \\
\text { lamento de retina. }\end{array}$ \\
\hline
\end{tabular}

Descritores: Miopia/complicações; Miopia/diagnóstico; Erros de refração; Retina; Degeneração macular; Fundus oculi
Trabalho realizado no Departamento de Oftalmologia do Hospital Universitário Clementino Fraga Filho da Universidade Federal do Rio de Janeiro - UFRJ - Rio de Janeiro (RJ) - Brasil.

${ }^{1}$ Residente do $3^{\circ}$ ano do Serviço de Oftalmologia do Hospital Universitário Clementino Fraga Filho da Universidade Federal do Rio de Janeiro - UFRJ - Rio de Janeiro (RJ) - Brasil.

${ }^{2}$ Residente do $3^{\circ}$ ano do Serviço de Oftalmologia do Hospital Universitário Clementino Fraga Filho da UFRJ - Rio de Janeiro (RJ) - Brasil.

${ }^{3}$ Médico do Setor de Retina e Vítreo do Serviço de Oftalmologia do Hospital Universitário Clementino Fraga Filho da UFRJ - Rio de Janeiro (RJ) - Brasil.

${ }^{4}$ Residente do $2^{\circ}$ ano do Serviço de Oftalmologia do Hospital Universitário Clementino Fraga Filho da UFRJ - Rio de Janeiro (RJ) - Brasil.

${ }^{5}$ Residente do $2^{\circ}$ ano do Serviço de Oftalmologia do Hospital Universitário Clementino Fraga Filho da UFRJ - Rio de Janeiro (RJ) - Brasil.

${ }^{6}$ Residente do $2^{\circ}$ ano do Serviço de Oftalmologia do Hospital Universitário Clementino Fraga Filho da UFRJ - Rio de Janeiro (RJ) - Brasil.

${ }^{7}$ Professor Adjunto Doutor, Chefe do Setor de Uveítes e Coordenador de Pós-Graduação do Departamento de Oftalmologia do Hospital Universitário Clementino Fraga Filho da UFRJ - Rio de Janeiro (RJ) - Brasil. Livre-Docente em Oftalmologia pela Universidade $\mathrm{Fe}$ deral de São Paulo - UNIFESP - São Paulo (SP) - Brasil. Endereço para correspondência: Oswaldo Ferreira Moura Brasil. Av. Epitácio Pessoa, 900/101 - Rio de Janeiro (RJ) CEP 22471-000

E-mail: dico@unisys.com.br

Recebido para publicação em 27.07.2004

Versão revisada recebida em 10.03.2005

Aprovação em 24.11.2005

Nota Editorial: Depois de concluída a análise do artigo sob sigilo editorial e com a anuência do Dr. Sérgio Lustosa da Cunha sobre a divulgação de seu nome como revisor, agradecemos sua participação neste processo.

\section{INTRODUÇÃO}

A miopia degenerativa, ou patológica, é uma importante causa de cegueira legal em países desenvolvidos ${ }^{(1-2)}$. Foi relatada prevalência de erro refrativo maior que - 7,9 dioptrias em 0,2 a 0,4\% da população nos Estados Unidos $^{(2)}$. No Japão, este valor chegou a $1 \%$ da população ${ }^{(3)}$.

Ela é caracterizada por erro refrativo de pelo menos -6,00 dioptrias com um comprimento axial do globo ocular de mais de $26 \mathrm{~mm}^{(4)}$. Duke-Elder define a miopia degenerativa como o tipo de miopia acompanhada por alterações degenerativas que ocorrem, particularmente, no segmento posterior do globo ocular ${ }^{(5)}$. Outra definição seria a alta miopia acompanhada por disfunção visual ${ }^{(3)}$.

A alta miopia é freqüentemente associada com prolongamento excessivo e progressivo do olho, resultando em uma variedade de alterações fundoscópicas, associadas com graus variáveis de perda visual ${ }^{(6)}$. Estas alterações acometem desde a fóvea até a periferia retiniana.

O objetivo deste estudo é avaliar a presença de alterações fundoscópicas em pacientes portadores de miopia degenerativa. 


\section{MÉTODOS}

A seleção dos pacientes para o estudo foi feita através de análise de prontuário de pacientes em acompanhamento oftalmológico ou com passagem pelo serviço de oftalmologia e diagnóstico de alta miopia (pelo menos $-6,00$ dioptrias). Os critérios de exclusão foram presença de catarata que justificasse baixa visual e história de cirurgia ocular prévia.

Foram selecionados 40 pacientes, sendo 10 homens e 30 mulheres, com idade variando de 9 a 72 anos e média de 43,6 anos. Todos foram submetidos a exame oftalmológico, incluindo refração subjetiva, biomicroscopia dos segmentos anterior e posterior e mapeamento da periferia retiniana, complementado com retinografia do pólo posterior e ecobiometria.

Após o exame completo, foram incluídos no estudo apenas os olhos que apresentaram erro refrativo de pelo menos $-6,00$ dioptrias com um comprimento axial do globo ocular de mais de $26 \mathrm{~mm}$.

\section{RESULTADOS}

Foram estudados 57 olhos de 37 pacientes, sendo 30 $(52,5 \%)$ direitos e $27(47,5 \%)$ esquerdos, com erro refrativo de $-6,25$ a $-28,50$ dioptrias, com média de $-14,05$, e comprimento axial de 26,06 a 32,86 mm, com média de 28,01. Entre os pacientes selecionados, 3 não tiveram seus olhos incluídos no estudo, pois o comprimento axial do globo era inferior a $26 \mathrm{~mm}$. Entre os pacientes estudados, 17 tiveram um dos olhos excluídos do estudo, sendo que destes olhos 11 não apresentavam miopia degenerativa, 4 apresentavam história de descolamento de retina, 1 era pseudofácico e 1 era afácico.

As alterações do disco óptico foram divididas em relação ao seu aspecto e a presença de crescente, temporal ou circunferencial. Não foram incluídos nesta avaliação 4 olhos de 3 pacientes, já que eram portadores de glaucoma. A alta freqüência de atrofia peripapilar e de crescente miópico dificulta e torna subjetiva a avaliação do aspecto do disco óptico. Notamos, no entanto, que a maioria dos pacientes apresentava discos ópticos ovais com maior diâmetro vertical e discos ópticos oblíquos.

O crescente temporal foi encontrado em 19 (36,5\%) olhos examinados e seu acometimento circunferencial em 11 (21\%) olhos.

A atrofia corio-retiniana, difusa ou localizada, foi um achado universal, apresentando-se em graus variados. Os vasos da coróide puderam ser visualizados em 20 (35\%) olhos. O estafiloma posterior foi encontrado em $6(10,5 \%)$ olhos, mancha de Fuchs em 2 (3,5\%) e "lacquer cracks" em apenas 1 $(1,5 \%)$ olho.

As figuras 1 e 2 mostram algumas alterações encontradas no pólo posterior.

O mapeamento da periferia retiniana evidenciou atrofia corio-retiniana tipo pavimentosa em 10 (17,5\%) olhos, branco sem pressão em $6(10,5 \%)$ olhos, degeneração "lattice" em 3
(5\%) olhos, ruptura retiniana em $2(3,5 \%)$ olhos e retinosquise em $1(1,5 \%)$ olho. Ambos os casos de ruptura retiniana foram encontrados em olhos com degeneração "lattice".

A tabela resume a freqüência das alterações fundoscópicas encontradas nos olhos examinados.

\section{DISCUSSÃO}

Pacientes com alongamento progressivo do globo ocular (miopia patológica) desenvolvem afinamento da coróide e epitélio pigmentar retiniano na área macular. Isto pode estar associado a disco óptico inclinado, atrofia corio-retiniana peripapilar, estafiloma posterior, áreas de atrofia do epitélio pigmentar retiniano e da coróide e "lacquer cracks" (7).

O disco óptico nos olhos com alta miopia é diferente dos

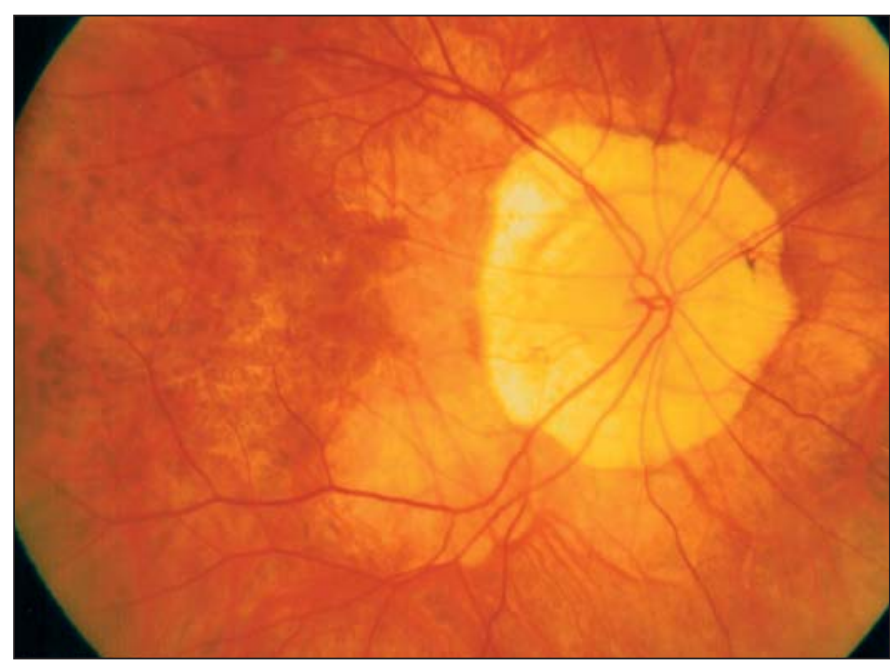

Figura 1 - Retinografia do olho direito de J.G.O., masculino, 62 anos, com $-11,25$ dioptrias e comprimento axial de $28,89 \mathrm{~mm}$. Nota-se crescente circunferencial e estafiloma posterior temporal inferior

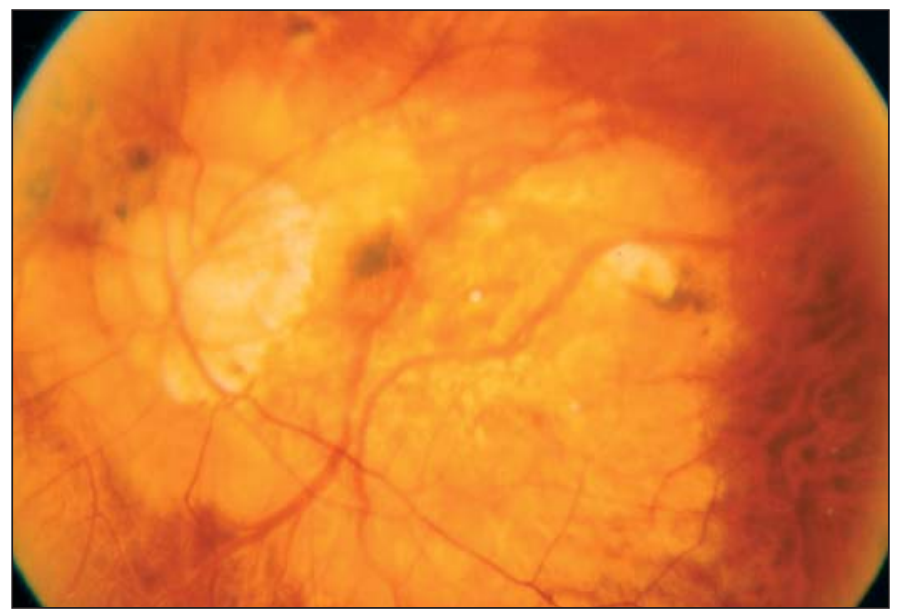

Figura 2 - Retinografia do olho esquerdo de N.C.S.F., feminina, 38 anos, com $-22,75$ dioptrias e comprimento axial de $32,39 \mathrm{~mm}$. Nota-se vizualização dos vasos coroidianos e manchas de Fuchs maculares 


\begin{tabular}{|lr|}
\hline $\begin{array}{l}\text { Tabela Freqüência de alterações fundoscópicas encontradas na } \\
\text { miopia patológica. Na freqüência de crescentes temporal e circun- } \\
\text { ferencial são excluidos quatro olhos com glaucoma }\end{array}$ \\
Alterações fundoscópicas & Olhos \\
Crescente temporal & $36,5 \%$ \\
Visualização dos vasos da coróide & $35 \%$ \\
Crescente circunferencial & $21 \%$ \\
Atrofia pavimentosa & $17,5 \%$ \\
Estafiloma posterior & $10,5 \%$ \\
Branco sem pressão & $10,5 \%$ \\
Degeneração "lattice" & $5 \%$ \\
Ruptura retiniana periférica & $3,5 \%$ \\
Mancha de Fuchs & $3,5 \%$ \\
"Lacquer cracks" & $1,5 \%$ \\
Retinosquise & $1,5 \%$ \\
\hline
\end{tabular}

discos ópticos normais, devendo ser considerado um macrodisco adquirido secundário, cujo tamanho é correlacionado com a refração e, possivelmente, com a idade ${ }^{(8)}$.

A atrofia corio-retiniana peripapilar é uma degeneração do complexo epitélio pigmentar retiniano e membrana de Bruch relacionada à idade ${ }^{(9)}$. Na miopia degenerativa, o primeiro sinal de tração tecidual é visto na margem temporal do disco óptico, onde é produzida uma área concêntrica de despigmentação, chamada crescente temporal, em que a esclera pode ser visua$\operatorname{lizada}^{(4)}$.

Os fatores que influenciam o desenvolvimento de atrofia corio-retiniana variam de acordo com a idade do paciente. Fatores locais, como o tamanho da membrana neovascular de coróide, determinam atrofia em jovens. Fatores sistêmicos, como a idade, têm importância maior em idosos ${ }^{(10)}$.

Em relação ao estafiloma posterior, Steidl e Pruett notaram que pacientes com estafilomas de menor profundidade apresentavam uma maior frequiência de membranas neovasculares de coróide, hemorragias e pior acuidade visual, sugerindo que o desenvolvimento de membranas neovasculares de coróide requer uma preservação relativa dos coriocapilares ${ }^{(11)}$. Estes autores encontraram uma prevalência de 79,5\% de estafilomas posteriores na análise de 116 olhos com erro refrativo miópico de, pelo menos, $-3,00$ dioptrias.

A mancha de Fuchs corresponde ao estágio final da neovascularização de coróide, sendo esta a mais comum complicação que afeta a visão na miopia degenerativa ${ }^{(12-13)}$.

"Lacquer cracks" representam rupturas cicatriciais e mecânicas do complexo epitélio pigmentar retiniano, membrana de Bruch e coriocapilar, com frequiência de 4,3\% em olhos alto míopes $^{(14)}$. Algus autores, encontraram "lacquer cracks" envolvendo a fóvea em $6 \%$ dos angiogramas realizados em pacientes com diagnóstico de miopia degenerativa ${ }^{(15)}$. Esta alteração pode estar associada a hemorragias sub-retinianas, que costumam apresentar curso benigno ${ }^{(16)}$.

O exame da periferia retiniana é especialmente importante nos olhos míopes, tendo em vista a maior frequiência de degeneração "lattice" nestes pacientes. Esta alteração é a mais importante lesão retiniana visível que predispõe a rupturas retinianas e descolamento de retina ${ }^{(17)}$.

Ao avaliar a periferia retiniana de pacientes míopes candidatos à cirurgia refrativa, alguns autores observaram que as alterações periféricas predisponentes ou não ao descolamento regmatogênico de retina apresentaram aumento de prevalência de acordo com o aumento do grau de miopia, com exceção das rupturas ${ }^{(18)}$.

Considerando o descolamento de retina, sabemos que a miopia é um fator de risco claramente relevante ${ }^{(19)}$. Foi demonstrado que $66,5 \%$ dos descolamentos regmatogênicos de retina ocorrem em míopes, sendo a razão da alta miopia sobre a miopia não-alta igual a $6^{(20)}$.

Nos pacientes estudados, $11 \%$ apresentavam história de descolamento de retina no olho contralateral, não incluído no estudo.

Em estudo de 308 olhos com miopia patológica enucleados, pós-operatórios e post mortem, Grossniklaus e Green encontraram atrofia corio-retiniana tipo pavimentosa em $14,3 \%$, degeneração "lattice" em 4,9\% e descolamento de retina em 11,4\% dos olhos examinados ${ }^{(21)}$. Estes dados apresentam valores muito parecidos com os obtidos em nosso estudo.

Apesar de não ser objetivo do estudo, consideramos relevante o achado de $8 \%$ dos pacientes estudados serem portadores de glaucoma, visto que os indivíduos míopes têm risco 2 a 3 vezes maior de desenvolver glaucoma quando comparados a indivíduos não-míopes ${ }^{(22)}$.

\section{CONCLUSÃO}

Consideramos que é freqüente a presença de alterações fundoscópicas em pacientes com miopia degenerativa e observamos que tais alterações podem, de forma variada, cursar com baixa visual. Devemos ter especial atenção nestes pacientes devido ao risco aumentado de descolamento de retina.

\section{ABSTRACT}

Purpose: To evaluate the presence of fundus changes in degenerative myopia. Methods: Forty patients with refractive error of at least -6.00 diopters were selected for ophthalmologic examination followed by posterior pole photograph and echobiometry. Results: We studied 57 eyes of 37 patients with refractive error ranging from -6.25 to -28.50 diopters, with mean -14.05 , and axial length ranging from 26.06 to $32.86 \mathrm{~mm}$, with mean 28.01. We found a temporal crescent in $36.5 \%$ and a peripapillary one in $20 \%$ of the eyes. Choroidal vessels were seen in $35 \%$ of the eyes. Posterior pole changes were as follow: posterior staphyloma in $10.5 \%$, Fuchs' spots in $3.5 \%$ and lacquer cracks in $1.5 \%$. Peripheral retinal examination revealed paving stone chorioretinal atrophy in $17.5 \%$, white without pressure in $10.5 \%$, lattice degeneration in 5\%, retinal tears in $3.5 \%$ and retinoschisis in $1.5 \%$ of the examined eyes. 
Conclusions: Fundus changes that lead to decreased vision are common in patients with degenerative myopia. Peripheral retinal examination is very important in these patients due to the increased risk of retinal detachment.

Keywords: Myopia/complications; Myopia/diagnosis; Refractive errors; Retina; Macular degeneration; Fundus oculi

\section{REFERÊNCIAS}

1. Ghafour IM, Allan D, Foulds WS. Common causes of blindness and visual handicap in the West of Scotland. Br J Ophthalmol. 1983;67(4):209-13.

2. Sperduto RD, Seigel D, Roberts J, Rowland M. Prevalence of myopia in the United States. Arch Ophthamol. 1983;101(3):405-7.

3. Tokoro T. On the definition of pathologic myopia in group studies. Acta Ophthalmol Suppl. 1988;185:107-8.

4. Soubrane G, Coscas G, Kuhn D. Myopia. In: Guyer DR, Yannuzzi LA, Chang S, Shields JA, Green WR, editors. Retina-vitreous-macula. Philadelphia: W. B. Saunders; 1999. p.189-205.

5. Duke-Elder S. Pathological refractive errors. In: Duke-Elder S, editor. System of ophthalmology. St. Louis: Mosby; 1970.

6. Tano Y. Pathologic myopia: where are we now? Am J Ophthalmol. 2002;134(5): 645-60.

7. Gass JDM. Stereoscopic atlas of macular diseases: diagnosis and treatment. St. Louis: Mosby; 1997.

8. Jonas LB, Gusek GC, Naumann GO. Optic disk morphometry in high myopia. Graefes Arch Clin Exp Ophthalmol. 1988;226(6):587-90.

9. Curcio CA, Saunders PL, Younger PW, Malek G. Peripapillary chorioretinal atrophy: Bruch's membrane changes and photoreceptor loss. Ophthalmology. 2000;107(2):334-43.
10. Kojima A, Ohno-Matsui K, Teramukai S, Yoshida T, Ishihara Y, Kobayashi $\mathrm{K}$, et al. Factors associated with the development of chorioretinal atrophy around choroidal neovascularization in pathologic myopia. Graefes Arch Clin Exp Ophthalmol. 2004;242(2):114-9.

11. Steidl SM, Pruett RC. Macular complications associated with posterior staphyloma. Am J Ophthalmol. 1997;123(2):181-7.

12. Hotchkiss ML, Fine SL. Pathologic myopia and choroidal neovascularization. Am J Ophthalmol. 1981;91(2):177-83.

13. Avila MP, Weiter JJ, Jalkh AE, Trempe CL, Pruett RC, Schepens CL. Natural history of choroidal neovascularization in degenerative myopia. Ophthalmology. 1984;91(12):1573-81.

14. Ohno-Matsui K, Tokoro T. The progression of lacquer cracks in pathologic myopia. Retina. 1996;16(1):29-37.

15. Vilela M, Nunes FO, Meyer GC, Germano C, Meyer RC. Estudo angiográfico da fóvea na miopia degenerativa. Rev Bras Oftalmol. 1992;51(3):373-8.

16. Shiokawa N, Grupenmacher F, Abib FC, Grupenmacher L. Lacquer crack e hemorragia subretiniana em míopes; um bom prognóstico visual. Rev Bras Oftalmol. 1991;50(1):40-3.

17. Wilkinson CP, Rice TA. Michels retinal detachment. St. Louis: Mosby; 1997.

18. Morales PHA, Farah ME, Höfling-Lima AL, Alleman N, Bonomo PP. Degenerações periféricas da retina em pacientes candidatos à cirurgia refrativa. Arq Bras Oftalmol. 2001;64(1):27-32.

19. Lavinsky J, Vargas JA, Fior O, Abujamra S. Lesões predisponentes e tratamento profilático do descolamento da retina. In: Moreira Jr. CA, Barsante C, Suzuki H, Lavinsky J, Gonçalves JOR, Nehemy MB, et al. Retina e vítreo: clínica e cirurgia. São Paulo: Roca; 2000. p.810-20.

20. Li X. Beijing Rhegmatogenous Retinal Detachment Study Group. Incidence and epidemiological characteristics of rhegmatogenous retinal detachment in Beijing, China. Ophthalmology. 2003;110(12):2413-7.

21. Grossniklaus HE, Green WR. Pathologic findings in pathologic myopia. Retina. 1992;12(2):127-33.

22. Mitchell P, Hourihan F, Sanbach J, Wang JJ. The relationship between glaucoma and myopia: the Blue Mountains Eye Study. Ophthalmology. 1999;106(10): 2010-5. Comment in: Ophthalmology. 2000;107(6):1026-7.

\section{Simpósio do Instifuto Penido Burnier}

\section{3 de Junhe de 2006}

\section{The Royal Palm Plaza Hotel Campinas - SP}

\section{tema: Relina Clínice e Cirúrgice}

